\title{
Virulent bacterial infection improves aversive learning performance in Drosophila melanogaster
}

\author{
Aurélie Babin, Sylvain Kolly and Tadeusz J. Kawecki \\ Department of Ecology and Evolution, University of Lausanne, Biophore, CH-1015 Lausanne, \\ Switzerland
}

Brain Behavior and Immunity 41:152-161 (2014)

doi: 10.1016/j.bbi.2014.05.008

With the exception of a few editorial chances in proofs, the content of this preprint is identical to the published version. The final published paper can be found on the publisher's website at http://www.sciencedirect.com/science/article/pii/S0889159114001305

Correspondence: tadeusz.kawecki@unil.ch

\begin{abstract}
Virulent infections are expected to impair learning ability, either as a direct consequence of stressed physiological state or as an adaptive response that minimizes diversion of energy from immune defense. This prediction has been well supported for mammals and bees. Here, we report an opposite result in Drosophila melanogaster. Using an odor-mechanical shock conditioning paradigm, we found that intestinal infection with bacterial pathogens Pseudomonas entomophila or Erwinia c. carotovora improved flies' learning performance after a $1 \mathrm{~h}$ retention interval. Infection with $P$. entomophila (but not E. c. carotovora) also improved learning performance after 5 min retention. No effect on learning performance was detected for intestinal infections with an avirulent GacA mutant of P. entomophila or for virulent systemic (hemocoel) infection with E. c. carotovora. Assays of unconditioned responses to odorants and shock do not support a major role for changes in general responsiveness to stimuli in explaining the changes in learning performance, although differences in their specific salience for learning cannot be excluded. Our results demonstrate that the effects of pathogens on learning performance in insects is less predictable than suggested by previous studies, and support the notion that immune stress can sometimes boost cognitive abilities.
\end{abstract}

Running headline: Infection and learning in fruit flies

Keywords: infection; intestinal pathogens; memory; insects; Drosophila melanogaster; psychoneuroimmunology 


\section{Introduction}

Cognitive processes are costly (Laughlin, 2001; Mery \& Kawecki, 2005) and highly sensitive to physiological stress, such as reduced oxygen or glucose supply (e.g., Warren \& Frier, 2005). Therefore, disease - and in particular infectious disease - is expected to adversely affect learning and memory, either as a direct consequence of abnormal physiological state or as an adaptive response that minimizes energy use for processes other than immune defense and maintenance of vital functions. On the other hand, it has been argued that stress (possibly including stress resulting from an infection) might boost cognitive abilities as part of an adaptive "fight or flight" response (Even et al., 2012; Roozendaal et al., 2009).

The majority of experimental data support the former view. Numerous studies in mice and rats found that infection with diverse live pathogens and parasites (Barrientos et al., 2006; Beraki et al., 2005; Cox \& Holland, 2001; Espinoza et al., 2013; Gibertini et al., 1995; Kavaliers et al., 1995) or stimulation of the immune system with foreign antigens (Cross-Mellor et al., 2009; Pugh et al., 1998; Shaw et al., 2001; Sparkman et al., 2005; Young et al., 2007) causes learning impairment in a variety of learning paradigms, although there are some exceptions (reviewed in Yirmiya \& Goshen, 2011). Analogous studies in insects have been limited to social bees and led to similar conclusions. After infection with their natural intestinal parasite Crithidia bombi, bumblebees exhibit impaired learning performance in a reward visual learning task (Gegear et al., 2006) and an operant motor learning task (Gegear et al., 2005). Infection of honeybees with the Deformed Wing Virus likewise impairs learning performance in an olfactory reward learning task (Iqbal \& Mueller, 2007). Furthermore, similar impairment in olfactory and visual learning tasks occurs in honeybees (Jaumann et al., 2013; Mallon et al., 2003) and bumblebees (Alghamdi et al., 2008; Riddell \& Mallon, 2006) whose immune system has been activated by injection of bacterial wall compounds (lipopolysaccharides). While most of the insect studies cited above relied on appetitive learning assays, one (Jaumann et al., 2013) has shown immune challenge-induced impairment in an aversive learning task. Thus, prevailing evidence indicates that infection with pathogens (and/or the resulting activation of the immune system) adversely affects learning performance of both mammals and insects.

Nevertheless, this conclusion is based on sterile bee workers which are unusual in that they have evolved to be efficient but expendable workforce for the colony rather than to maximize their own lifetime reproductive success. Thus, their responses to stress and the associated trade-offs may not necessarily be representative of the non-social majority of insect species. This calls for studies on the effects of infection on learning in non-social insects.

In this paper we report on the effects of infection with bacterial pathogens on aversive olfactory learning performance in Drosophila melanogaster, a favorite system for studying the neural mechanisms of associative learning (Davis, 2005). We investigated the effects of intestinal infection (acquired by feeding), as well as systemic infection (sepsis induced by inoculating bacteria into the flies' hemocoel). While the latter is a classic way of studying infection and immunity in insects, the former is of greater ecological significance as it is the natural way in which bacterial pathogens are acquired by fruit flies (Lemaitre \& Hoffmann, 2007). For the intestinal infection, we used two natural Gram-negative bacterial pathogens: the highly virulent generalist entomopathogen Pseudomonas entomophila $(\mathrm{Pe})$ and the mildly virulent opportunistic pathogen Erwinia c. carotovora (Ecc). To study the effect of systemic infection on learning performance we used Ecc inoculation into the hemocoel, which under our procedures resulted in comparably high virulence and similar amplitude of immune response as intestinal infection with Pe. Aversive learning performance was assessed with a Pavlovian conditioning assay which quantified avoidance of an odor (conditioned stimulus, CS) previously associated with aversive mechanical shock (unconditioned stimulus, US), with 5 min and 1 
$\mathrm{h}$ retention times. In addition, to test for differences in perception or salience of the stimuli, we tested the unconditioned response to the odorants used as CS and the responsiveness to mechanical shock used as the US.

\section{Materials and methods}

\subsection{Fly and bacterial strains}

Our outbred stock population of D. melanogaster was derived from more than 200 mated female flies caught in Valais (Switzerland). The population had been maintained under standard laboratory conditions $\left(25{ }^{\circ} \mathrm{C}, 60 \%\right.$ relative humidity, 12:12 light: dark cycle, standard yeast-sucrose-cornmeal food medium); the experiments began after it had been in the lab for 6 months. Flies were maintained at a maximum density of 250 eggs per $30 \mathrm{ml}$ of food. In all experiments we used mated females aged 3 to 7 days from adult eclosion that were collected under $\mathrm{CO}_{2}$ anesthesia and allowed to recover for 24 hours (with food) before the infection treatments.

All bacterial strains were kindly provided by Bruno Lemaitre (EPFL, Lausanne, Switzerland). The virulent strain of Pseudomonas entomophila (Pe), isolated in Guadeloupe (Vodovar et al., 2005), leads to strong activation of the immune system in the gut and the fat body, extensive gut damage, and high mortality over the few days following infection (Buchon et al., 2009a; Liehl et al., 2006; Vodovar et al., 2005). The gacA mutant strain of P. entomophila (GacA), derived from the virulent strain, is avirulent upon intestinal infection and does not elicit immune response in the gut (Vodovar et al., 2005). Erwinia c. carotovora (Ecc; strain 15; Basset et al., 2000) multiplies in the gut, induces some gut damage, and elicits an immune response, but causes negligible mortality in otherwise healthy flies and is eventually cleared from the gut (Basset et al., 2000). Bacteria were grown at $28{ }^{\circ} \mathrm{C}$ in standard Luria-Bertani broth (10 g Bactotryptone ${ }^{\circledR}, 5 \mathrm{~g}$ Bactoyeast ${ }^{\circledR}, 10 \mathrm{~g} \mathrm{NaCl} ; 1000 \mathrm{ml}$ distilled water). After culture centrifugation $\left(3000 \mathrm{rpm}, 20 \mathrm{~min}, 4{ }^{\circ} \mathrm{C}\right)$, the optical density of the bacterial pellet was adjusted to $\mathrm{OD}_{600 \mathrm{~nm}} \approx 200\left(\approx 10^{11}\right.$ cells $\left./ \mathrm{ml}\right)$ with sterile $0.9 \%$ saline buffer. Heat-killed bacteria were obtained by heating the bacterial pellet at $100{ }^{\circ} \mathrm{C}$ for $5 \mathrm{~min}$.

\subsection{Infection treatments}

For intestinal infection, flies were first transferred in groups of 30 to empty vials for 2 hours to motivate them to feed. Subsequently, they were transferred to vials with an agar medium $(22 \mathrm{~g} / \mathrm{l})$ covered with a filter disk soaked with $140 \mu \mathrm{l}$ of an infective mixture. The infective mixture contained, in equal proportions, $5 \%$ sucrose and bacterial pellet (Pe, GacA, or Ecc; final optical density on the filter disk $\mathrm{OD} \approx 100$ ) for infection treatments; for the saline control we used a 50:50 mixture of $5 \%$ sucrose and sterile $0.9 \%$ saline buffer. Flies were maintained in these vials under standard laboratory conditions for $20 \mathrm{~h}$. Feeding assays with dyed food mixture confirmed that virtually all flies ingested bacteria during this oral infection treatment. All behavioral and gene expression assays were carried out immediately after the end of the infection period.

For systemic infection, flies were anesthetized under $\mathrm{CO}_{2}$ and pricked on the side of the thorax with a needle (diameter $150 \mu \mathrm{m}$ ) dipped into saline buffer for a control treatment (saline), live Ecc, dead (heat-killed) Ecc or dead (heat-killed) Pe, adjusted at the same OD as for intestinal infection (except where specified otherwise). After injection, flies were maintained under standard laboratory conditions for $20 \mathrm{~h}$ before the assays. 


\subsection{Virulence and immune response}

Before the behavioral assays, we assessed virulence of these pathogens and the degree to which they elicit an immune response in our Drosophila strain under the conditions used in the behavioral assays. To assess the virulence of the pathogens upon intestinal or systemic infection, we quantified mortality of infected flies. Five replicate groups of 30 female flies were infected intestinally or systemically as described in section 2.2. Subsequently they were transferred to a fresh vial with food. Dead flies were counted twice a day (morning and afternoon) for 4 days counting from the onset of the infection treatment.

To assess the activation of the immune system, we used quantitative reverse-transcription PCR (RTqPCR) to quantify the expression of the Diptericin gene, coding for an antimicrobial peptide regulated by the Imd signaling pathway (Lemaitre \& Hoffmann, 2007). Twenty hours from the onset of intestinal infection or from inoculation by pricking, 10 flies per biological replicate were collected under $\mathrm{CO}_{2}$ anesthesia, and immediately frozen in liquid nitrogen. After sample homogenization, total RNA was extracted using Qiazol ${ }^{\circledR}$ (Qiagen) and chloroform. cDNA was produced from $1 \mu \mathrm{g}$ of total RNA with Superscript II (Life Technologies), diluted 1/20 in Tris buffer, and subsequently used for quantitative real-time PCR with SYBR Green I (Roche) on an ABI 7900HT cycler (Applied Biosystems), with three technical replicates per biological sample. In addition to Diptericin (primers: sense 5' GCT GCG CAA TCG CTT CTA CT 3', anti-sense 5' TGG TGG AGT GGG CTT CAT G 3'; Buchon et al., 2009a) we amplified three reference genes, Rpl32 (sense 5' GAC GCT TCA AGG GAC AGT ATC TG 3', anti-sense 5' AAA CGC GGT TCT GCA TGA G 3'; Buchon et al., 2009b), Actin5c (sense 5' CAA GTG CGA GTG GTG GAA GTT 3' , anti-sense 5' GCA GGT GGT TCC GCT CTT T 3'; Colombani et al., 2005) and Efl $\alpha$ (sense 5' GTG AAG CAG CTG ATC GTT GGT 3', anti-sense 5' CAT AAC GGG CCT CGC TGT AT 3'; Vijendravarma et al., 2012). For each biological replicate, the expression of Diptericin was normalized to the geometric mean expression of the reference genes with $\mathrm{qBase}{ }^{\mathrm{PLUS}}$ software (Biogazelle; Hellemans et al., 2007). The resulting Normalized Relative Quantities were log-transformed for the analysis.

\subsection{Effect of infection on aversive learning}

To address the main question of this paper we assessed the learning performance of flies subject to the above infection treatments in an olfactory Pavlovian conditioning assay, which quantified the avoidance of an odorant previously associated with aversive mechanical shock (Mery \& Kawecki, 2005; Mery et al., 2007). This assay was performed on groups of 30 flies, comprising the basic unit of replication. For conditioning, these groups of flies were transferred to test tubes with perforated ends, connected to a system of flexible tubes for odorant and air delivery, and fixed on a test tube shaker (vortex VV3, VWR) for shock delivery. Conditioning consisted of two massed (i.e., back-to-back) cycles. In each cycle, flies were first exposed for $30 \mathrm{~s}$ to one odorant (CS+, i.e. the odorant flies are conditioned to avoid) and simultaneously subject to mechanical shocks (1 s pulses at $2000 \mathrm{rpm}$ every 5 s), followed by $60 \mathrm{~s}$ of humid air, $30 \mathrm{~s}$ of the second odorant (CS-), without shock, and another $60 \mathrm{~s}$ period of humid air. The odorants were delivered by percolating air through odorants dissolved in paraffin oil. Subsequently, the flies were tested for their choice between the two odorants in an elevator T-maze and their choice was recorded after $60 \mathrm{~s}$. The test was carried out either 5 min (which is as soon as practically possible) or $1 \mathrm{~h}$ after the end of conditioning. The $5 \mathrm{~min}$ response presumably reflects short-term memory while the $1 \mathrm{~h}$ response is thought to rely mostly on middle-term memory with a possible contribution of so-called anesthesia-resistant memory (Davis, 2005). In most assays the odorants were 4-methylcyclohexanol (MCH, $0.6 \mathrm{ml} / \mathrm{l})$ and 3-octanol (OCT, $0.4 \mathrm{ml} / \mathrm{l})$; however, to 
test if the results are robust to the odorants used as CS, in one experiment we used an alternative odorant pair, amyl acetate (AA, $0.4 \mathrm{ml} / \mathrm{l})$ and benzaldehyde (BA, $0.6 \mathrm{ml} / \mathrm{l})$.

Following a convention used in olfactory aversive learning in Drosophila (e.g., Mery \& Kawecki, 2005; Schwaerzel et al., 2003; Waddell \& Quinn, 2001), learning performance was quantified as a learning index equal to the difference between the proportion of flies choosing odor $A$ when odor $B$ is $\mathrm{CS}+$ and the proportion choosing odor $A$ when odor $A$ is CS+. Thus, the learning index quantifies the combined effects of how well the animal learns and how well it retains the learned information until the test, while controlling for an overall relative preference towards one odorant of the pair. Each replicate value of learning index was calculated by pairing replicates collected from the same culture vial and conditioned concurrently in opposite directions. Flies that remained in the center of the Tmaze (i.e., chose neither odor) were excluded from the calculation of the proportions. A positive index means avoidance of $\mathrm{CS}+$, i.e. of the odorant previously coupled with mechanical shock; the maximum learning index is 1 . We also analyzed learning index values calculated with arcsine-square-roottransformed proportions and obtained virtually identical results, so only analysis on non-transformed data is reported.

The effect of intestinal infection treatments on learning performance was investigated in four separate experiments; the first three experiments used $\mathrm{MCH}$ and OCT as odorants, the fourth used the alternative odorants $\mathrm{AA}$ and $\mathrm{BA}$. The first and the fourth experiments included all four intestinal infection treatments (saline, Pe, GacA and Ecc); for logistic reasons the Ecc treatment was not performed in the second experiment, and the GacA treatment was absent from the third. The effect of systemic infection was studied in two replicate experiments, both including all four treatments (saline, live Ecc, dead Ecc, dead Pe). Based on a trend suggested by these first two experiments (see Results), a third experiment was carried out to test specifically the effect of systemic infection with dead Pe on 1 hour memory, but using a greater dose $(\mathrm{OD} \approx 400)$.

\subsection{Unconditioned responses to odorants}

Flies subject to the four intestinal infection treatments were tested in the T-maze for their unconditioned absolute and relative preferences for the odorants used in the learning assays described above. The absolute preference assay involved choice between a single odorant and the solvent; the relative preference assay involved choice between the two odorants of a pair ( $\mathrm{MCH}$ versus OCT and AA versus BA). Flies were subject to the same mechanical shock as in learning assays (see above) but in the absence of odorants; this was done to account for potential sensitization effects of shock. Five minutes later flies were transferred to the T-maze and given $1 \mathrm{~min}$ to make their choice. The absolute preference was measured as the proportion of flies choosing the odorant over the solvent, the relative preference as the proportion of flies choosing the first odorant of the pair. Analysis on nontransformed and arcsine-square root-transformed proportions yielded virtually identical results, so we only report the former.

\subsection{Responsiveness to mechanical shock}

To assess the effect of the infection treatments on the reactivity to mechanical shock (i.e., the US) we used a locomotor startle assay, which measured the effect of shock on locomotor activity. Petri dishes (diameter $60 \mathrm{~mm}$ ) were fixed horizontally on a test-tube shaker. Flies were transferred individually to the Petri dishes without anesthesia and allowed to acclimatize for $7 \mathrm{~min}$. Subsequently, fly behavior was video-recorded for $3 \mathrm{~min}$ (baseline pre-shock activity) before flies received two $1 \mathrm{~s}$ pulses of 
mechanical shock identical to that used in the learning assay, and then were recorded for another 3 min (post-shock activity). Distances covered by flies during these pre- and post-shock 3 min periods were estimated with the tracking software cvMob 2.0 (http://www.cvmob.ufba.br/). Locomotion consisted of walking, running and jumps; the low ceiling in the Petri dish effectively prevented flies from flying. Additionally, from the same recordings we estimated the duration of the last complete bout of activity immediately before shock and the first bout of locomotion immediately after shock (defined to be terminated when the fly stopped for at least $1 \mathrm{~s}$ ), as well as the distance covered during those locomotion bouts. All flies responded to shock by moving but about $5 \%$ did not move during the preshock phase of the recording and were treated as missing data for the bout analysis. The duration of activity bout had a positively skewed distribution and was log-transformed before analysis, which normalized the distribution of residuals. Two experimental blocks of this assay have been performed for the four intestinal infection treatments. Because we found an effect of virulent intestinal infection on the post-shock locomotor bout duration (see Results), we also performed this assay (one block) for the systemic infection treatments in order to see if such effects are specific to intestinal infection.

\subsection{Statistical analysis}

Statistical analyses were performed with JMP 8.0 and SAS 9.3 (SAS Institute Inc.). A proportional hazard Cox regression was fitted to survival data. As pre- and post-shock distances measured in the locomotor startle assay were highly skewed distributions and no simple transformation made the residual normal, these data were analyzed using non-parametric Wilcoxon and Kruskal-Wallis tests. ANOVA was used for other response variables, transformed as indicated above. Normality of residuals was verified with normal quantile plots (Q-Q plots). The fixed effects included infection treatment and, where appropriate, odorant (in absolute odor preference assay) or pre-shock versus post-shock activity period (for the shock responsiveness assay). Where data from several replicate experiments were analyzed together (i.e. for the assays of learning and of locomotor response to shock), the experiment was treated as block effect. In all analyses, interactions were included in initial models and are reported as appropriate, but non-significant interactions were excluded from the final models used to test for main effects. The analysis of the effect of intestinal infection of learning employed type 4 sum-of-squares (SAS Institute Inc., 1989) to account for missing treatment $\times$ experiment combinations (see above). The results of the learning assays were visualized by plotting the means and standard errors of each treatment $\times$ experiment combination as well as least-square mean estimates for each treatment obtained in the ANOVA, these estimates are corrected for the design being unbalanced. Pairwise comparisons between treatments were carried out using planned contrasts within the ANOVA; the critical P-values were adjusted with sequential Bonferroni correction (Rice, 1989) to account for the six possible pairwise contrasts between treatments. For conclusions that depended on non-rejection of a null hypothesis we provided $95 \%$ confidence intervals for the corresponding (non-significant) effect size as a measure of confidence in the negative result (Hoenig \& Heisey, 2001).

\section{Results}

\subsection{Virulence and immune response}

In order to assess the virulence and immune system activation resulting from our infection treatments, we quantified mortality and whole-body expression of the antimicrobial peptide Diptericin, a standard readout for the Imd pathway involved in immune defense against Gram-negative bacteria (Lemaitre \& Hoffmann, 2007). As previously reported for other Drosophila strains (Buchon et al., 2009b; Liehl et 
al., 2006; Vodovar et al., 2005), intestinal infection with the virulent strain of $P$. entomophila (Pe) led to substantial mortality, with $35 \%$ of flies dying within four days of infection, whereas virtually no flies fed an avirulent $P$. entomophila mutant (GacA) or the saline buffer died (Fig 1A). Intestinal Pe infection upregulated the expression of Diptericin 81-fold; in contrast, the GacA mutant induced only a moderate 3.7-fold upregulation of Diptericin (Fig. 1B). Flies intestinally infected with Erwinia c. carotovora (Ecc) showed negligible mortality, indistinguishable from the saline control (Fig 1A). However, they did show markedly (11-fold) elevated Diptericin expression (Fig. 1B).

Systemic infection with Ecc (by pricking flies with bacteria-coated needle) resulted in about $50 \%$ mortality within four days (Fig 1C). This treatment also increased the expression of Diptericin 20-fold (Fig. 1D). In contrast, systemic inoculation with dead (heat-killed) Ecc or dead Pe was associated with very low mortality, not distinguishable from saline-pricked flies (Fig. 1C), although it induced a moderate increase in expression of Diptericin (Fig. 1D).

Based on the above results, we decided to carry out the behavioral assays reported below at $20 \mathrm{~h}$ postinfection, before any mortality due to infection occurred (Fig. 1A,C). This assured that the results were not biased by survival of infection being non-random with respect to the assayed traits (This would not have been possible for a systemic infection with live Pe, which caused substantial mortality well before $20 \mathrm{~h}$; A. Babin unpublished data). While the focus was on the highly virulent infection treatments (intestinal with Pe and systemic with Ecc), intestinal infection with Ecc was also included as an example of mildly virulent intestinal infection, whereas systemic treatments with dead Ecc and dead Pe were regarded as emulating a mild systemic immune challenge.

\subsection{Effect of intestinal infection on aversive learning}

The effects of intestinal infection on olfactory aversive learning were tested in four separate experiments. Despite considerable variation in the overall learning performance among the experiments, the differences between intestinal infection treatments were highly consistent across experiments, including experiment 4, which used different odorants than experiments 1-3 (Fig. 2A,B; for statistical tests see figures and figure legends). For both retention intervals, the mean learning index of flies infected with virulent Pe was higher than that of saline controls (Fig. 2A,B). The increase in learning performance of flies infected with Pe tended to be proportionally greater at $1 \mathrm{~h}$ than at $5 \mathrm{~min}(26 \%$ versus $13 \%)$. Flies infected with the avirulent GacA mutant of P. entomophila performed virtually identically to saline controls, indicating that the effect of Pe infection on learning performance was driven by its virulence (Fig. 2A,B). Flies intestinally infected with the mildly virulent Ecc showed $21 \%$ better learning performance than saline controls for $1 \mathrm{~h}$ retention (Fig. 2B), but were indistinguishable from the controls for 5 min retention (Fig. 2A).

\subsection{Effect of systemic infection on aversive learning}

In contrast to intestinal infection, systemic infection treatments had no significant effect on learning performance (Fig. 3A,B). In particular, systemic infection treatment with live Ecc (which was as virulent as intestinal infection with Pe; Fig. 1) was indistinguishable from the saline control $(95 \%$ confidence interval for the effect size for both retention intervals combined: [ $0.083,0.064]$ ). The results did suggest a trend for systemic infection with dead Pe to improve performance at $1 \mathrm{~h}$ retention compared to the saline control (Fig. 3B). To verify this apparent effect, we performed an additional experiment with a higher dose $(\mathrm{OD} \approx 400)$ of dead Pe; this experiment did not confirm the trend (not 
illustrated, learning index mean \pm SEM: dead Pe $0.67 \pm 0.03$, Saline $0.74 \pm 0.04 ; F_{1,29}=2.2, P=0.15$, $N=16)$.

\subsection{Unconditioned response to odorants}

We found no evidence that the infection treatments affected unconditioned responses to the odorants used in the learning assays. As is usually the case in this learning paradigm (e.g., Mery et al., 2007), in the absence of conditioning all odorants used in this study were moderately aversive, i.e. chosen over solvent by less than $50 \%$ of flies in the absolute preference test. The degree of aversion differed among odorants, but was not affected by intestinal infection (Fig. 4A). Likewise, intestinal infection had no effect on the relative preference of flies choosing between odorants of either pair (Fig. 4B). Thus, the differences in learning performance reported above are unlikely to be mediated by enhanced perception or higher salience of odorants.

\subsection{Responsiveness to mechanical shock}

Flies responded to mechanical shock by a marked increase in locomotor activity, which subsequently gradually declined but was still clearly elevated in the third minute after shock (Fig. 5A). However, the intestinal infection treatments did not affect the distance covered within the last 3 min preceding the shock, within the first minute after shock, or within the first 3 min after shock (Fig. 5A).

However, the first bout of locomotion immediately following the shock was about $50 \%$ longer in Peinfected flies than in saline controls (Fig. 5B). In contrast, Ecc-infected flies did not differ from saline controls in this measure of shock response (Fig. 5B). No differences were detected among the intestinal infection treatments in the duration of the last complete bout of locomotion before the shock (Fig. 5B). The distance covered during the first bout of locomotion showed a similar pattern (Fig. 5C). Thus, the fact that Pe-infected flies covered the same distance in the first minute after shock (Fig. 5A) in spite of their first locomotion bout being longer implies that they moved less after the locomotion bout.

Like the intestinal infection treatments, the systemic infection treatments had no effect on the distance covered within the 3 min before and after shock (Figure 6A), nor on the length of the last pre-shock locomotion bout (Figure 6B,C). However, the systemic infection treatments affected the duration of, and the distance covered during, the first bout of locomotion after shock (Figure 6B,C). Specifically, in all three treatments involving bacteria the first bout of locomotion was shorter than in the saline controls, although for the dead Pe treatment the difference was only marginally significant (Fig. 6B).

\section{Discussion}

Contrary to expectations based on previous studies in insects, Drosophila infected with intestinal pathogens showed enhanced aversive olfactory learning compared to uninfected controls. The effect was moderate but reproducible across experiments, and it was observed both for the highly virulent Pseudomonas entomophila and the mildly virulent Erwinia c. carotovora. For P. entomophila infection, the stronger learned response was observed both 5 minutes and 1 hour after conditioning; for E. c. carotovora only 1 hour after. By contrast, flies infected with the avirulent gacA mutant of $P$. entomophila exhibited learning performance similar to uninfected controls. This indicates that the improvement in learning performance following intestinal infection is mediated by virulence of the pathogens, possibly in interaction with the immune response. 
The effects on learning were limited to intestinal infections; systemic infection by injection of live $E$. c. carotovora in the hemocoel did not improve learning performance even though such infection induces a strong immune response and is at least as virulent as intestinal infection with $P$. entomophila. This dependence of the learning effects on the route of infection is reminiscent of findings in rodents which suggest a similar dependence of learning effects on the mode of administration of immune system elicitors (Yirmiya \& Goshen, 2011). In insects, the effects of different routes of infection or different pathogens on learning were apparently never compared in a single study. However, different studies found impaired learning resulting from intestinal infections with a natural trypanosomid pathogen (Gegear et al., 2005; Gegear et al., 2006), systemic infection with a virus (Iqbal \& Mueller, 2007), and systemic injections of an immune system elicitor (Alghamdi et al., 2008; Jaumann et al., 2013; Mallon et al., 2003). Thus, the finding of learning impairment upon infection or immune system stimulation in bees seems rather robust to the type of immune challenge and the route of its administration. We can only speculate about the causes of the differential effect of intestinal versus systemic infection in our study. The immune response to intestinal and systemic infection with Gram-negative bacteria is largely mediated by the same pathways (in particular the Imd pathway), although expressed in different tissues (gut epithelium versus fat body) (Lemaitre \& Hoffmann, 2007). However, systemic immunity involves an important cellular component whereas gut immunity relies more heavily on ROS production and proliferation of intestinal stem cells to repair the gut epithelium damaged by the infection (Lemaitre \& Hoffmann, 2007). Irrespective of the reason for this difference, it should be noted that intestinal infection with ingested pathogens is the most common way of acquiring bacterial pathogens in Drosophila (Buchon et al., 2013). We therefore focus the remainder of the discussion on the effects of intestinal infections.

A stronger response to conditioning could be mediated by a greater sensitivity to the unconditioned stimulus (US), in this case mechanical shock (Rescorla, 1988). Because flies respond to shock by several-fold increase in locomotion (presumably trying to escape the danger signified by shock), we assessed shock sensitivity in terms of a locomotor response. We found that flies suffering from intestinal infection with $P$. entomophila moved for longer than uninfected controls during the first bout of locomotion after shock. This suggests that they may perceive the shock as a somewhat stronger stimulus, which could have partly contributed to their better learning performance. However, other results do not support a relationship between the first post-shock locomotion bout and learning performance. First, intestinal infection with E.c. carotovora had no effect on this locomotor response to shock, even though it also improved learning performance, albeit only for the $1 \mathrm{~h}$ memory retention interval. Second, the post-shock locomotion bout in flies that were systemically infected with live or dead E.c. carotovora was half as long as that in saline controls, yet there was no corresponding difference in learning performance. Finally, despite the differences in the first locomotor bout duration, the total distance covered in the first minute or the first three minutes post-shock was not affected by the infection treatments, even though the agitation induced by shock persisted over this interval. This suggests that the post-shock locomotion bout duration may not be highly relevant as a measure of sensory perception of shock. Altogether, these results do not support the role of differences in general shock sensitivity as a major factor explaining the effects of intestinal infection on learning performance observed in our study.

However, this does not preclude the possibility that the improvement of learning performance upon intestinal infection might have resulted from an increase in specific salience of shock as US in the learning process (rather than general sensitivity to shock). As one possibility, this might be mediated by strengthened synaptic connections between the neurons that carry the US information and the mushroom body Kenyon cells, which integrate US and olfactory CS information in insects (Davis, 2005). Although the neurons conveying the mechanical shock US to mushroom bodies are unknown, 
flies perceive mechanical shock similarly to electric shock (Acevedo et al., 2007), and electric shock US is transmitted to mushroom bodies by dopaminergic neurons (Aso et al., 2010; Schwaerzel et al., 2003). While we have no evidence for involvement of dopamine in our results, dopamine is a plausible candidate link between immune system and learning, as discussed below.

Irrespective of the possible role of US sensitivity or salience, some aspects of our results suggest that intestinal pathogenic infection in Drosophila may improve memory retention. In particular, in flies infected intestinally with the mildly virulent E. c. carotovora, an improved response to conditioning was only observed for $1 \mathrm{~h}$ memory retention interval. At $5 \mathrm{~min}$ following conditioning the performance of infected flies was virtually identical to saline-fed controls, suggesting the same degree of initial learning. Better memory retention is also suggested by the greater proportional effect of $P$. entomophila infection on $1 \mathrm{~h}$ than on 5 min performance (26\% versus $13 \%)$. Behavioral responses assayed $1 \mathrm{~h}$ after odor-shock conditioning in Drosophila are thought to rely mostly on middle-term memory (Davis, 2005), possibly with a contribution of so-called anesthesia-resistant memory (Isabel et al., 2004). Interestingly, memory retention in Drosophila is sensitive to some aspects of physiological state that do not affect immediate responses to conditioning based on short-term memory. For example, middle-term memory declines with age earlier than short-term memory (Tamura et al., 2003), an effect which can be specifically reversed by a mutation (Yamazaki et al., 2007). Long-term memory formation is specifically downregulated upon starvation (Placais \& Preat, 2013). Furthermore, mild dietary restriction has been shown to boost $1 \mathrm{~h}$ aversive memory retention in young flies with no effect on immediate conditioning response (Burger et al., 2010). This last finding coupled with the results presented here support the notion that mild physiological stress may under some circumstances improve middle-term and/or anesthesia-resistant memory retention in Drosophila.

That stress may boost memory formation and retention is well-established in mammals (reviewed in Roozendaal et al., 2009). In particular, several rodent studies show improved learning performance under immune stress, mediated at least in part by proinflamatory cytokines (reviewed in Yirmiya \& Goshen, 2011). Interestingly, in Drosophila, temporary downregulation of the cytokine-activated JAK/STAT pathway in mushroom bodies (learning centers in insect brain) leads to impairment of long-term aversive memory, although no consistent effects on unconsolidated memory have been found (Copf et al., 2010). Another candidate for the molecular mechanism mediating cross-talk between immunity and learning in insects are catecholamines. The stress response in insects involves increasing levels of dopamine and octopamine in the brain (reviewed by Even et al., 2012). Dopamine is also involved in immune response against parasitoids and eukaryotic parasites (Nappi \& Christensen, 2005; Sideri et al., 2008) and in epithelial response to bacterial infection (Davis \& Engstrom, 2012). At the same time, dopamine and octopamine play a central role in aversive learning, in particular in relaying the US signal (Aso et al., 2010; Claridge-Chang et al., 2009; Schwaerzel et al., 2003) and general arousal (Van Swinderen \& Andretic, 2011); systemic pharmacological treatments with dopamine can enhance aversive learning (Agarwal et al., 2011). Thus, physiological response to stress in general (and infection in particular) might enhance cognitive performance of insects via increases in the levels of dopamine and octopamine, a prediction formulated by Even et al. (2012).

While our results are consistent with this prediction, they are in apparent contradiction to studies on the effects of infection or immune challenge on learning performance in honey and bumble bees, all of which report learning impairment after infection or immune challenge (Alghamdi et al., 2008; Gegear et al., 2005; Gegear et al., 2006; Iqbal \& Mueller, 2007; Jaumann et al., 2013; Mallon et al., 2003; Riddell \& Mallon, 2006). Assuming no publication bias, the prevalence of negative effects in the literature suggests that adverse consequences of immune stress usually outweigh any potential beneficial effects of the stress response on learning acquisition and memory retention. This is the case in mammals, where negative effects of immune stress on learning are commonly found whereas 
positive effects are rare and sensitive to experimental details (Yirmiya \& Goshen, 2011). We can only speculate about the reasons for the apparent discrepancy between our results and the bee studies cited above. Although most of the bee studies relied on appetitive learning assays, Jaumann et al. (2013) showed that immunochallenged bees perform poorly in a Pavlovian aversive paradigm involving an association between odors and electric shock. Thus, the difference between those studies and our results is not likely to be explainable by appetitive versus aversive nature of learning task, or by the sensory channels involved, especially given that Drosophila apparently perceive electric and mechanical shock as similar stimuli (Acevedo et al., 2007). Another possibility is the nature of infection - none of the bee studies involved intestinal infection with live bacterial pathogens. However, several studies found impaired learning in bees injected with lipopolysaccharides, which are components of the cell wall of Gram-negative bacteria (Alghamdi et al., 2008; Jaumann et al., 2013; Mallon et al., 2003; Riddell \& Mallon, 2006). Our systemic treatments with heat-killed bacteria likewise inoculated the hemolymph with lipopolysaccharides, yet, they did not affect the learning performance of the flies despite activating immune defense.

The third potential explanation points to differences between the study species. Although the basic mechanisms of immunity and learning are evolutionarily conserved between flies and bees (Fischman et al., 2011; Himmelreich \& Gruenewald, 2012), they are likely to differ in details. For example, flies possess significantly more immune genes than bees (Fischman et al., 2011), and bee mushroom bodies are structurally more complex than those of flies (Farris \& Schulmeister, 2011). Such differences may reflect idiosyncrasies of their respective phylogenetic history. However, bees differ ecologically from most insects in being eusocial - they live in dense colonies which share food, facilitating spread of pathogens, and they seem to rely on learning more heavily than most other insects (Chittka \& Skorupski, 2011; Müller, 2002). Perhaps most importantly, the bee studies were carried out on workers, which are effectively sterile and optimized by evolution for their work for the colony rather than to maximize lifetime reproductive output. Thus, for example, energetic trade-offs between immune or stress response and cognitive performance might be more acute in worker bees (Jaumann et al., 2013), which do not have the option to reallocate energy from reproduction. Consistent with this notion, moderate nutritional stress reduces aversive olfactory learning in honey bees (Jaumann et al., 2013) but has no effect on short-term memory and a positive effect on 1 hour memory retention in Drosophila (Burger et al., 2010). Whether there is indeed a general distinction between how immune stress affects learning performance in social hymenoptera versus in non-social insects requires more studies on the latter. In the meantime, our study demonstrates that the effects of pathogens on learning performance in insects are more complex and less predictable than previously thought.

\section{Acknowledgements}

We thank B. Lemaitre, N. A. Broderick and S. Chakrabarti (EPFL, Lausanne) for bacterial strains and advice, R. K. Vijendravarma for advice, M. Zini and J. S. Burger for help with experiments, the Department of Fundamental Microbiology (University of Lausanne), the Lausanne Genomic Technologies Facility for technical support, and B. Hollis, D. Naug and three anonymous referees for comments. This work has been supported by the Swiss National Science Foundation.

\section{References}

Acevedo, S. F., Froudarakis, E. I., Kanellopoulos, A. , Skoulakis, E. M. C., 2007. Protection from premature habituation requires functional mushroom bodies in Drosophila. Learn. Memory 14, 376384.doi:10.1101/1m.566007. 
Agarwal, M., Guzman, M. G., Morales-Matos, C., Diaz, R. A. D., Abramson, C. I. , Giray, T., 2011. Dopamine and octopamine influence avoidance learning of honey bees in a place preference assay. PLoS ONE 6.doi:e 25371

10.1371/journal.pone.0025371.

Alghamdi, A., Dalton, L., Phillis, A., Rosato, E. , Mallon, E. B., 2008. Immune response impairs learning in free-flying bumble-bees. Biology Letters 4, 479-481.doi:10.1098/rsbl.2008.0331.

Aso, Y., Siwanowicz, I., Bracker, L., Ito, K., Kitamoto, T., Tanimoto, H., 2010. Specific Dopaminergic Neurons for the Formation of Labile Aversive Memory. Current Biol. 20, 14451451.doi:10.1016/j.cub.2010.06.048.

Barrientos, R. M., Higgins, E. A., Biedenkapp, J. C., Sprunger, D. B., Wright-Hardesty, K. J., Watkins, L. R., Rudy, J. W. , Maier, S. F., 2006. Peripheral infection and aging interact to impar hippocampal memory consolidation. Neurobiol. Aging 27, 723-732.

Basset, A., Khush, R. S., Braun, A., Gardan, L., Boccard, F., Hoffmann, J. A. , Lemaitre, B., 2000. The phytopathogenic bacteria Erwinia carotovora infects Drosophila and activates an immune response. Proc. Natl. Acad. Sci. USA 97, 3376-3381.

Beraki, S., Aronsson, F., Karlsson, H., Ogren, S. O. , Kristensson, K., 2005. Influenza A virus infection causes alterations in expression of synaptic regulatory genes combined with changes in cognitive and emotional behaviors in mice. Mol. Psychiatr. 10, 299-308.

Buchon, N., Broderick, N. A., Chakrabarti, S. , Lemaitre, B., 2009a. Invasive and indigenous microbiota impact intestinal stem cell activity through multiple pathways in Drosophila. Genes \& Development 23, 23332344.doi:10.1101/gad.1827009.

Buchon, N., Broderick, N. A. , Lemaitre, B., 2013. Gut homeostasis in a microbial world: insights from Drosophila melanogaster. Nat. Rev. Microbiol. 11, 615-626.doi:10.1038/nrmicro3074.

Buchon, N., Broderick, N. A., Poidevin, M., Pradervand, S. , Lemaitre, B., 2009b. Drosophila intestinal response to bacterial infection: activation of host defense and stem cell proliferation. Cell Host Microbe 5, 200211.doi:10.1016/j.chom.2009.01.003.

Burger, J. M. S., Buechel, S. D. , Kawecki, T. J., 2010. Dietary restriction affects lifespan but not cognitive aging in Drosophila melanogaster. Aging Cell 9, 327-335.

Chittka, L. , Skorupski, P., 2011. Information processing in miniature brains Introduction. Proc. R. Soc. B 278, 885-888.doi:10.1098/rspb.2010.2699.

Claridge-Chang, A., Roorda, R. D., Vrontou, E., Sjulson, L., Li, H. Y., Hirsh, J., Miesenbock, G., 2009. Writing Memories with Light-Addressable Reinforcement Circuitry. Cell 139, 405415.doi:10.1016/j.cell.2009.08.034.

Colombani, J., Bianchini, L., Layalle, S., Pondeville, E., Dauphin-Villemant, C., Antoniewski, C., Carre, C., Noselli, S. , Leopold, P., 2005. Antagonistic actions of ecdysone and insulins determine final size in Drosophila. Science 310, 667-670.doi:10.1126/science.1119432.

Copf, T., Goguel, V., Lampin-Saint-Amaux, V., Scaplehorn, N. , Preat, T., 2010. Cytokine signaling through the JAK/STAT pathway is required for long-term memory in Drosophila. Proc. Natl. Acad. Sci. USA 108, 8059-8064.doi:10.1073/pnas.1012919108.

Cox, D. M. , Holland, C. V., 2001. Relationship between three intensity levels of Toxocara canis larvae in the brain and effects on exploration, anxiety, learning and memory in the murine host. J. Helminthol. 75, $33-41$

Cross-Mellor, S. K., Foley, K. A., Parker, L. A. , Ossenkopp, K. P., 2009. Lipopolysaccharide dose dependently impairs rapid toxin $(\mathrm{LiCl})$-induced gustatory conditioning: A taste reactivity examination of the conditioned taste aversion. Brain Behav. Immun. 23, 204-216.doi:10.1016/j.bbi.2008.09.006.

Davis, M. M. , Engstrom, Y., 2012. Immune response in the barrier epithelia: lessons from the fruit fly Drosophila melanogaster. Journal of Innate Immunity 4, 273-283.doi:10.1159/000332947.

Davis, R. L., 2005. Olfactory memory formation in Drosophila: From molecular to systems neuroscience. Annu. Rev. Neurosci. 28, 275-302. 
Espinoza, J. A., Bohmwald, K., Cespedes, P. F., Gomez, R. S., Riquelme, S. A., Cortes, C. M., Valenzuela, J. A., Sandoval, R. A., Pancetti, F. C., Bueno, S. M., Riedel, C. A. , Kalergis, A. M., 2013. Impaired learning resulting from Respiratory Syncytial Virus infection. Proc. Natl. Acad. Sci. USA 110, 91129117.doi:10.1073/pnas.1217508110.

Even, N., Devaud, J. M. , Barron, A. B., 2012. General stress responses in the honey bee. Insects 3, 1271-1298.

Farris, S. M. , Schulmeister, S., 2011. Parasitoidism, not sociality, is associated with the evolution of elaborate mushroom bodies in the brains of hymenopteran insects. Proc. R. Soc. B 278, 940951.doi:10.1098/rspb.2010.2161.

Fischman, B. J., Woodard, S. H. , Robinson, G. E., 2011. Molecular evolutionary analyses of insect societies. Proc. Natl. Acad. Sci. USA 108, 10847-10854.doi:10.1073/pnas.1100301108.

Gegear, R. J., Otterstatter, M. C. , Thomson, J. D., 2005. Does parasitic infection impair the ability of bumblebees to learn flower-handling techniques? Anim. Behav. 70, 209-215.

Gegear, R. J., Otterstatter, M. C. , Thomson, J. D., 2006. Bumble-bee foragers infected by a gut parasite have an impaired ability to utilize floral information. Proc. R. Soc. B 273, 1073-1078.

Gibertini, M., Newton, C., Friedman, H. , Klein, T. W., 1995. Spatial learning impairment in mice infected with Legionella pneumophila or administered exogenous interleukin-1-B. Brain Behav. Immun. 9, 113-128.

Hellemans, J., Mortier, G., De Paepe, A., Speleman, F. , Vandesompele, J., 2007. qBase relative quantification framework and software for management and automated analysis of real-time quantitative PCR data. Genome Biol. 8.doi:R19

10.1186/gb-2007-8-2-r19.

Himmelreich, S. , Gruenewald, B., 2012. Cellular physiology of olfactory learning in the honeybee brain. Apidologie 43, 308-321.doi:10.1007/s13592-012-0135-Z.

Hoenig, J. M. , Heisey, D. M., 2001. The abuse of power: the pervasive fallacy of power calculations for data analysis. The American Statistician 55, 19-24.

Iqbal, J., Mueller, U., 2007. Virus infection causes specific learning deficits in honeybee foragers. Proc. R. Soc. B 274, 1517-1521.doi:10.1098/rspb.2007.0022.

Isabel, G., Pascual, A. , Preat, T., 2004. Exclusive consolidated memory phases in Drosophila. Science 304, 1024-1027.

Jaumann, S., Scudelari, R. , Naug, D., 2013. Energetic cost of learning and memory can cause cognitive impairment in honeybees. Biology Letters 9, 20130149.

Kavaliers, M., Colwell, D. D. , Galea, L. A. M., 1995. Parasitic Infection Impairs Spatial-Learning in Mice. Anim. Behav. 50, 223-229.

Laughlin, S. B., 2001. Energy as a constraint on the coding and processing of sensory information. Curr. Opin. Neurobiol. 11, 475-480.

Lemaitre, B. , Hoffmann, J., 2007. The host defense of Drosophila melanogaster. Annual Review of Immunology 25, 697-743.doi:10.1146/annurev.immunol.25.022106.141615.

Liehl, P., Blight, M., Vodovar, N., Boccard, F. , Lemaitre, B., 2006. Prevalence of local immune response against oral infection in a Drosophila/Pseudomonas infection model. PLoS Pathogens 2, 551561.doi:e56.

Mallon, E. B., Brockmann, A. , Schmid-Hempel, P., 2003. Immune response inhibits associative learning in insects. Proc. R. Soc. B 270, 2471-2473.

Mery, F., Kawecki, T. J., 2005. A cost of long-term memory in Drosophila. Science 308, 1148-1148.

Mery, F., Pont, J., Preat, T. , Kawecki, T. J., 2007. Experimental evolution of olfactory memory in Drosophila melanogaster. Physiol. Biochem. Zool. 80, 399-405.

Müller, U., 2002. Learning in honeybees: from molecules to behaviour. Zoology 105, 313320.doi:10.1078/0944-2006-00075.

Nappi, A. J. , Christensen, B. M., 2005. Melanogenesis and associated cytotoxic reactions: Applications to insect innate immunity. Insect Biochemistry and Molecular Biology 35, 443459.doi:10.1016/j.ibmb.2005.01.014. 
Placais, P. Y. , Preat, T., 2013. To favor survival under food shortage, the brain disables costly memory. Science 339, 440-442.doi:10.1126/science.1226018.

Pugh, C. R., Kumagawa, K., Fleshner, M., Watkins, L. R., Maier, S. F. , Rudy, J. W., 1998. Selective Effects of Peripheral Lipopolysaccharide Administration on Contextual and Auditory-Cue Fear Conditioning. Brain Behav. Immun. 12, 212-229.

Rescorla, R. A., 1988. Behavioral-Studies of Pavlovian Conditioning. Annu. Rev. Neurosci. 11, 329-352.

Rice, W. R., 1989. Analyzing tables of statistical tests. Evolution 43, 223-225.

Riddell, C. E. , Mallon, E. B., 2006. Insect psychoneuroimmunology: Immune response reduces learning in protein starved bumblebees (Bombus terrestris). Brain Behavior and Immunity 20, 135-138.

Roozendaal, B., McEwen, B. S. , Chattarji, S., 2009. Stress, memory and the amygdala. Nat. Rev. Neurosci. 10, 423-433.doi:10.1038/nrn2651.

SAS Institute Inc., 1989. SAS/STAT user's guide, version 6, fourth edition. SAS Institute, Cary, NC.

Schwaerzel, M., Monastirioti, M., Scholz, H., Friggi-Grelin, F., Birman, S. , Heisenberg, M., 2003. Dopamine and octopamine differentiate between aversive and appetitive olfactory memories in Drosophila. J. Neurosci. 23, 10495-10502.

Shaw, K. N., Commins, S. , O'Mara, S. M., 2001. Lipopolysaccharide causes deficits in spatial learning in the watermaze but not in BDNF expression in the rat dentate gyrus. Behav. Brain Res. 124, 47-54.

Sideri, M., Tsakas, S., Markoutsa, E., Lampropoulou, M. , Marmaras, V. J., 2008. Innate immunity in insects: surface-associated dopa decarboxylase-dependent pathways regulate phagocytosis, nodulation and melanization in medfly haemocytes. Immunology 123, 528-537.doi:10.1111/j.1365-2567.2007.02722.x.

Sparkman, N. L., Kohman, R. A., Garcia, A. K. , Boehm, G. W., 2005. Peripheral lipopolysaccharide administration impairs two-way active avoidance conditioning in C57BL/6J mice. Physiol. Behav. 85, 278-288.

Tamura, T., Chiang, A. S., Ito, N., Liu, H. P., Horiuchi, J., Tully, T. , Saitoe, M., 2003. Aging specifically impairs amnesiac-dependent memory in Drosophila. Neuron 40, 1003-1011.

Van Swinderen, B., Andretic, R., 2011. Dopamine in Drosophila: setting arousal thresholds in a miniature brain. Proc. R. Soc. B 278, 906-913.doi:10.1098/rspb.2010.2564.

Vijendravarma, R. K., Narasimha, S. , Kawecki, T. J., 2012. Evolution of foraging behaviour in response to chronic malnutrition in Drosophila melanogaster. Proc. R. Soc. B 279, 3540-3546.

Vodovar, N., Vinals, M., Liehl, P., Basset, A., Degrouard, J., Spellman, P., Boccard, F. , Lemaitre, B., 2005. Drosophila host defense after oral infection by an entomopathogenic Pseudomonas species. Proc. Natl. Acad. Sci. USA 102, 11414-11419.

Waddell, S. , Quinn, W. G., 2001. What can we teach Drosophila? What can they teach us? Trends Genet. 17 , 719-726.

Warren, R. E. , Frier, B. M., 2005. Hypoglycaemia and cognitive function. Diabetes, Obesity and Metabolism 7, 493-503.

Yamazaki, D., Horiuchi, J., Nakagami, Y., Nagano, S., Tamura, T. , Saitoe, M., 2007. The Drosophila DCO mutation suppresses age-related memory impairment without affecting lifespan. Nature Neuroscience 10, 478-484.

Yirmiya, R., Goshen, I., 2011. Immune modulation of learning, memory, neural plasticity and neurogenesis. Brain Behav. Immun. 25, 181-213.doi:10.1016/j.bbi.2010.10.015.

Young, E. E., Baumbauer, K. M., Elliot, A., Joynes, R. L., 2007. Lipopolysaccharide induces a spinal learning deficit that is blocked by IL-1 receptor antagonism. Brain Behav. Immun. 21, 748-757. 


\section{FIGURES}
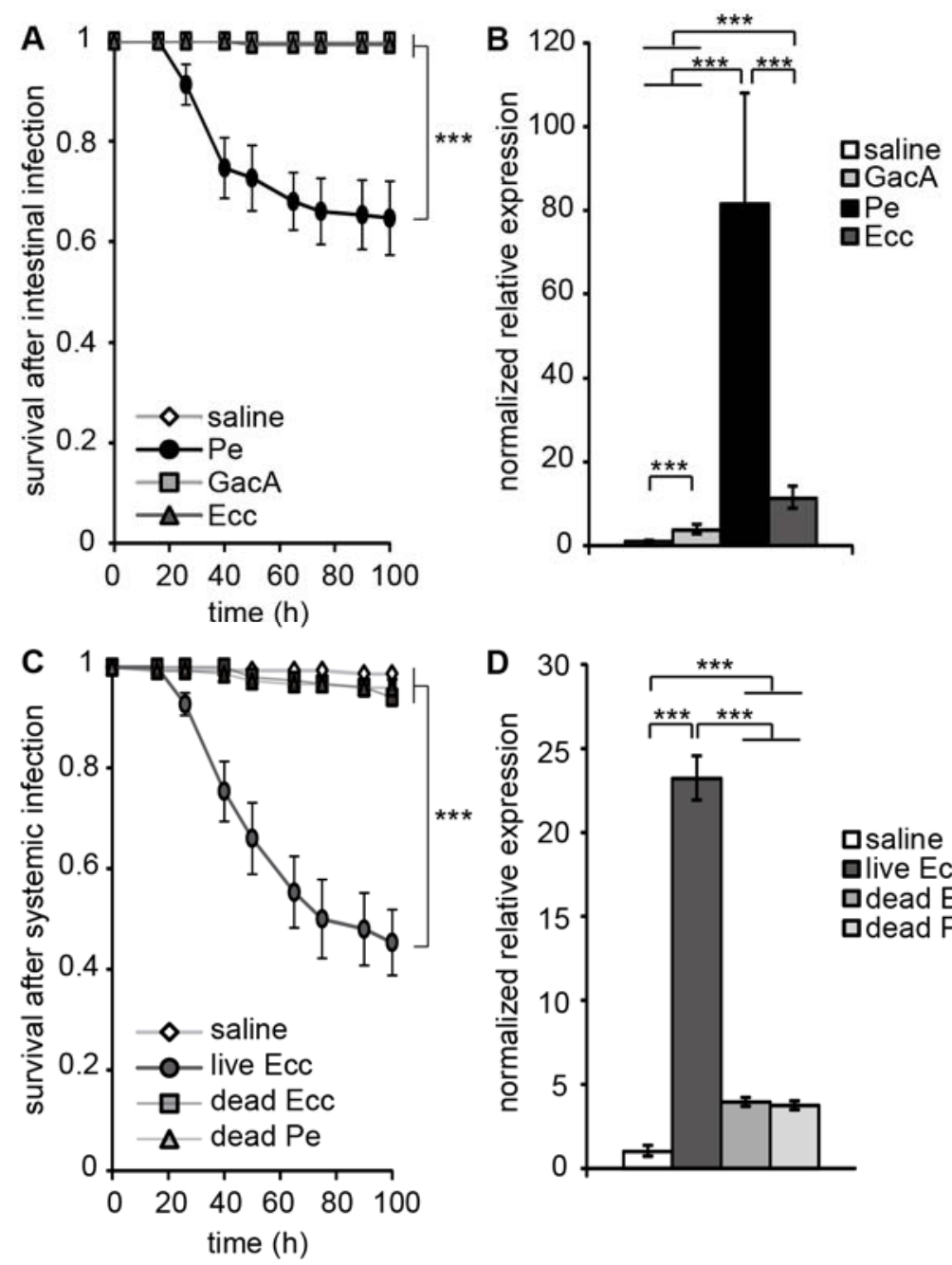

Figure 1. Virulence and activation of the immune system by experimental infection treatments. (A) Probability of survival and $(B)$ relative expression of the Imd-dependent antimicrobial peptide Diptericin in flies subject to intestinal infection with one of three live bacterial strains (Pe, Ecc, or GacA) or fed the saline buffer. In panel $(A)$ the saline, GacA and Ecc treatments result in virtually no mortality so the lines are not distinguishable. (C) Survival and (D) relative Diptericin expression after the systemic infection treatments. Symbols are means \pm SE (back-transformed from the log scale for the relative expression). $N=5$ replicates of 30 flies each per treatment in the survival assays; $N=6$ biological replicates of 10 flies for quantitative RT-PCR. 
A

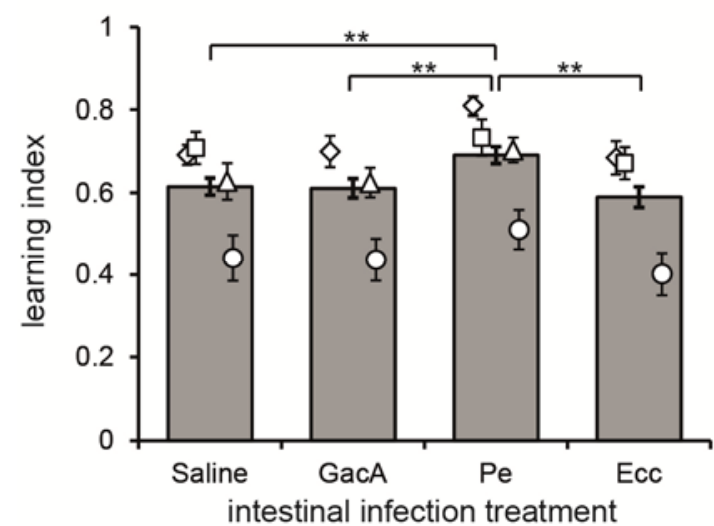

B $1 \mathrm{~h}$ retention

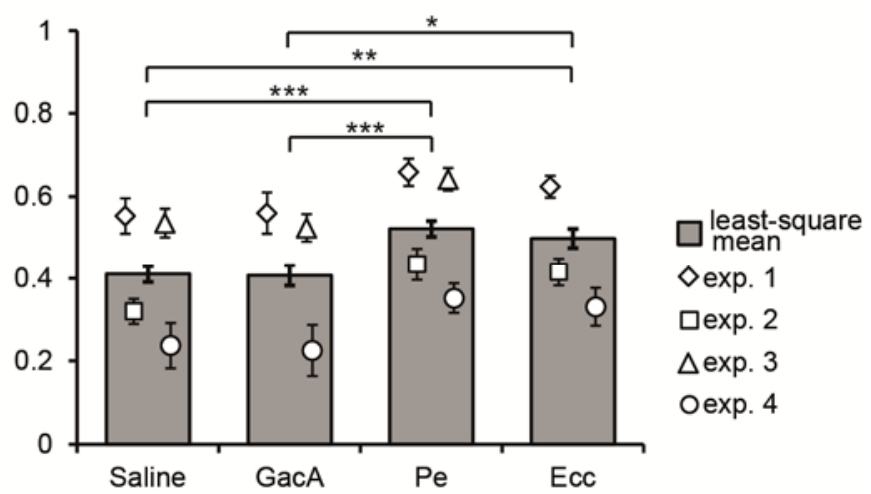

intestinal infection treatment

Figure 2. The effects of intestinal infection treatments on aversive olfactory learning performance. Symbols indicate means of four experiments (blocks), the bar plot shows overall least-square means for each treatment. Error bars correspond to \pm 1 SEM. ${ }^{*} P<0.02,{ }^{* *} P<0.01$, ${ }^{* *} P<0.001$; all significant pairwise comparisons stand after sequential Bonferroni correction. (A) 5 min memory retention; ANOVA: treatment $F_{3,201}=4.4, P=0.0053$, experiment $F_{3,201}=34.8, P=0.0053$; treatment $\times$ experiment $F_{7,194}=0.2, P=0.99$. (B) $1 \mathrm{~h}$ memory retention; ANOVA: treatment $F_{3,261}=7.9, P<0.0001$, experiment $F_{3,261}=48.7, P<0.0001$; treatment $\times$ experiment $F_{7,254}=$ $0.1, P=0.99$. Sample sizes: experiment $1, N=15-16$ indices per treatment, experiment $2, N=10-11$ for 5 min retention, $N=30-31$ for $1 \mathrm{~h}$ retention, experiment $3, N=18-20$, experiment $4 N=14$; each replicate index has been obtained from two groups of 30 flies conditioned in opposite direction. For logistic reasons GacA treatment was absent in experiment 2; Ecc treatment was absent in experiment 3.
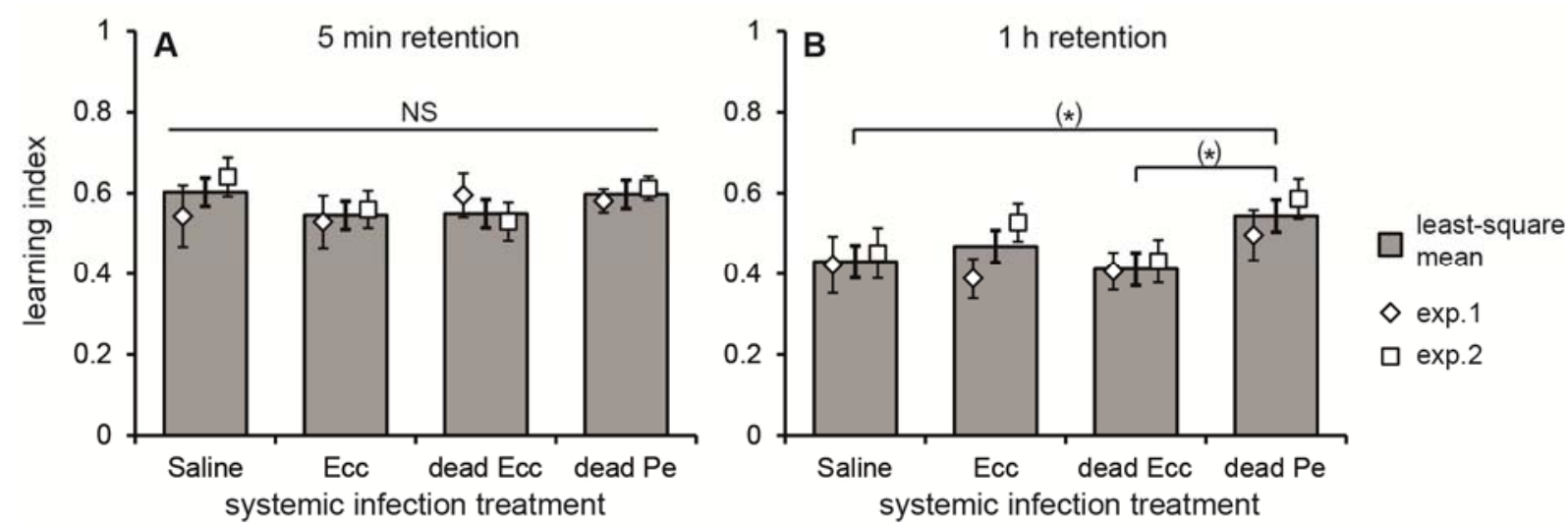

Figure 3. The effects of systemic infection treatments on aversive olfactory learning performance. Symbols indicate means of two experiments (blocks), bars show least-square means for each treatment; error bars correspond to \pm 1 SEM. (A) 5 min memory retention; ANOVA: treatment $F_{3,87}=0.8, P=0.51$, experiment $F_{1,87}=$ $0.4, P=0.52$; treatment $\times$ experiment $F_{3,84}=0.8, P=0.48$. (B) $1 \mathrm{~h}$ memory retention; ANOVA: treatment $F_{3,87}=$ 2.2, $P=0.096$, experiment $F_{1,87}=2.7, P=0.095$; treatment $\times$ experiment $F_{3,84}=0.4, P=0.73 ;{ }^{*} 0.02<\mathrm{P}<0.05$, non-significant after sequential Bonferroni correction. Sample sizes: experiment $1 \mathrm{~N}=8$, experiment $2 \mathrm{~N}=15$. 

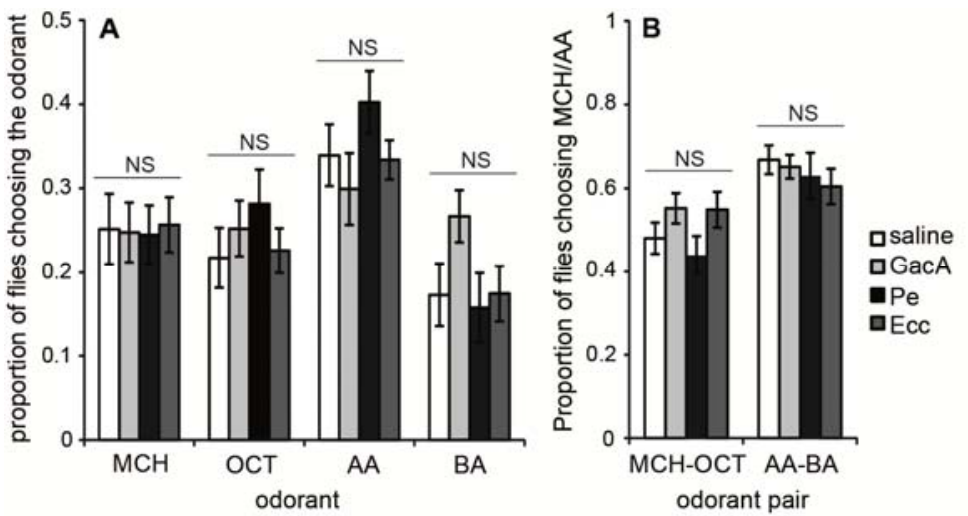

Figure 4. Unconditioned preferences for odorants used in the learning assays shown by flies previously subject to the intestinal infection treatments. Plots represent means \pm 1 SEM of proportions of flies making a particular choice. "NS": all $P \geq 0.14$. (A) Preference for an odorant versus solvent; sample sizes: $N=16$ proportions per treatment for $\mathrm{MCH}$ and $\mathrm{OCT}, N=12$ for $\mathrm{AA}$ and $\mathrm{BA}$. (B) Relative preference shown by flies facing a choice between two odorants; the odorant pairs $\mathrm{MCH}-\mathrm{OCT}$ and AA-BA are the same as used in the learning assays; sample sizes: $N=15$ for $\mathrm{MCH}$ versus $\mathrm{OCT}, N=12$ for $\mathrm{AA}$ versus $\mathrm{BA}$.
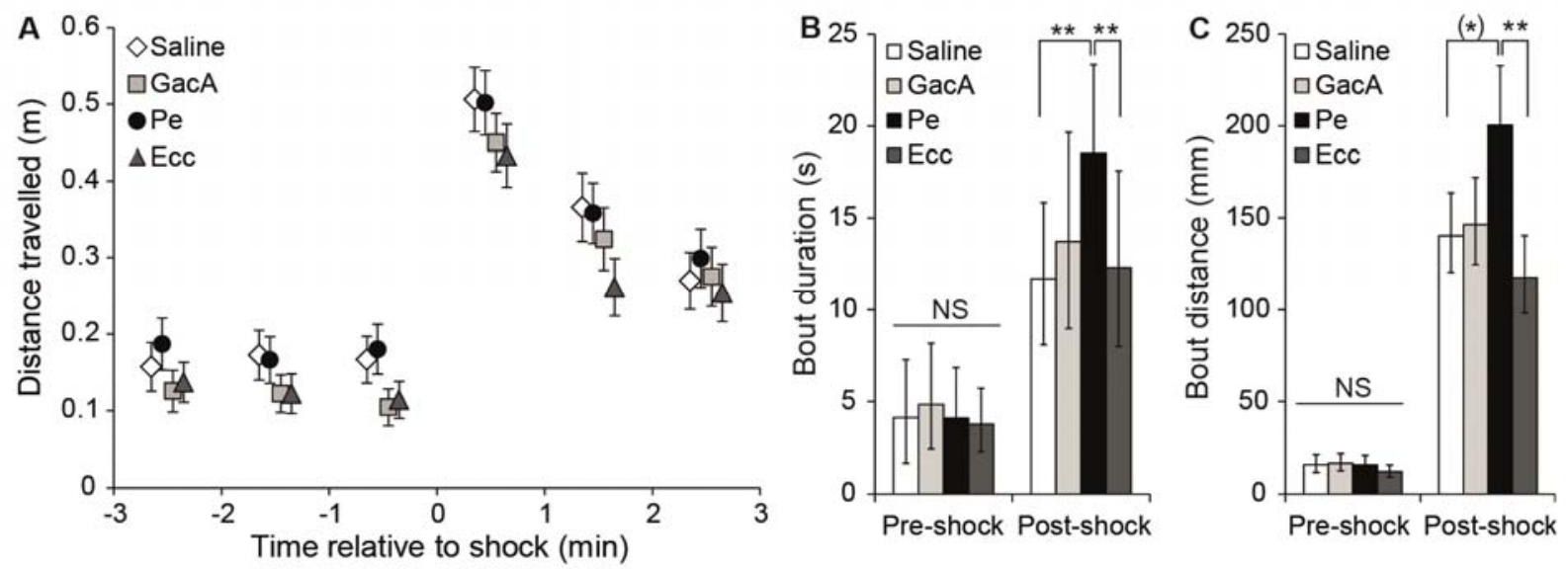

Figure 5. The effects of intestinal infection treatments on locomotor response to mechanical shock (used as the unconditioned stimulus in the learning assays). Individual flies were recorded for $3 \mathrm{~min}$, subject to shock, and recorded for another $3 \mathrm{~min}$ ( $N=56-58$ flies per treatment). (A) Locomotor activity quantified as the average distance $( \pm$ SEM) covered per minute by flies before and after mechanical shock. Kruskal-Wallis test for the effect of treatment on the distance covered: 3 min pre-shock $x^{2}=0.8$, d.f. $=3, P=0.84 ; 3$ min after shock $x^{2}=3.0$, d.f. $=$ $3, P=0.38$; the $1^{\text {st }}$ minute after shock $x^{2}=2.5$, d.f. $=3, P=0.47$. (B) Duration of and (C) distance covered during the last bout of locomotion before shock and the first bout of locomotion after shock (until the fly stopped for at least $1 \mathrm{~s}$ ). ${ }^{* *} P<0.01 ;{ }^{*} P<0.05$ not significant after sequential Bonferroni correction. Error bars correspond to \pm 1 SEM. The assay was performed in two experimental blocks which differed in overall level of activity, but the treatment effects were consistent across blocks (all treatment $\times$ block interactions $P>0.2$ ). 

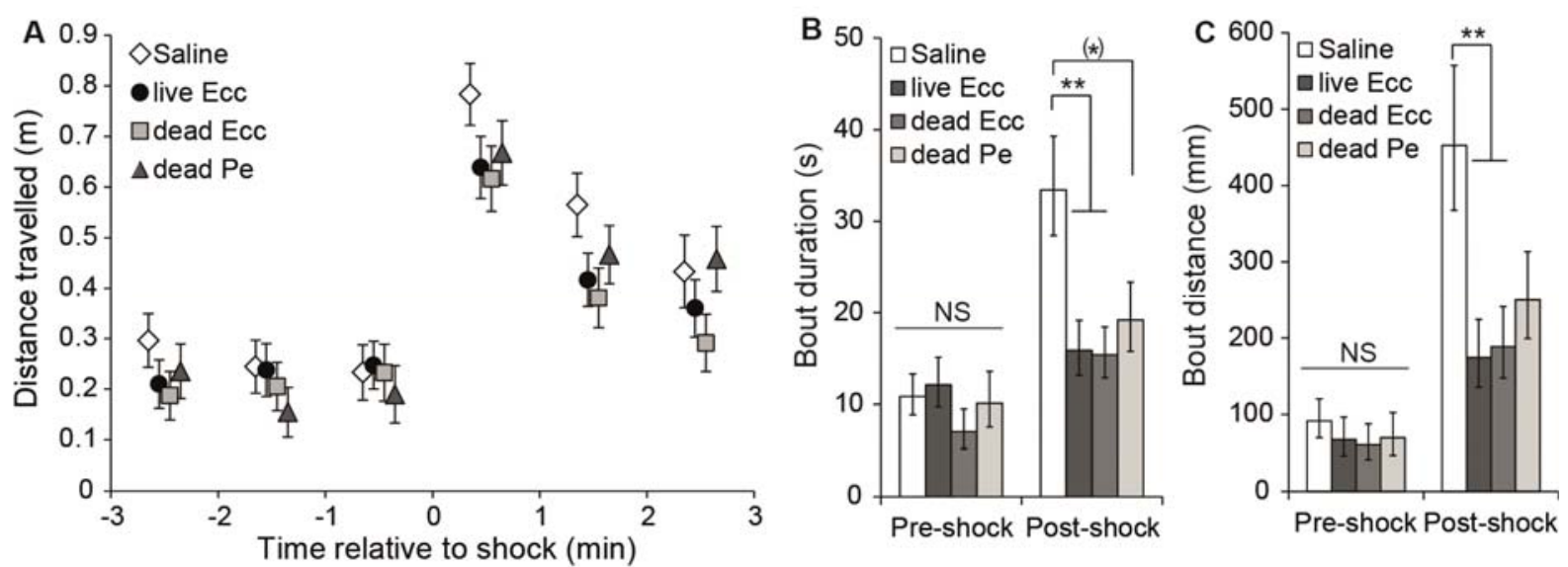

Figure 6. The effects of systemic infection on responsiveness to mechanical shock, assayed as in Figure 5 (means $\pm \mathrm{SEM}$ ). $N=23-25$ flies per treatment. (A) Average distance covered per minute before and after mechanical shock. ANOVA, treatment effect: pre-shock $F_{1,109}=0.3, P=0.81$, post-shock $F_{1,109}=1.7, P=0.16$.

(B) Duration of and (C) distance covered during the last pre-shock and the first post-shock bout of locomotion. ${ }^{* *} P$ $<0.01 ;{ }^{(*)} P<0.05$ not significant after sequential Bonferroni correction. 